\title{
Conditions for preserving negative imaginary properties in feedback interconnections and an application to multi- agent systems \\ DOI:
}

10.1109/MED.2017.7984271

\section{Document Version}

Accepted author manuscript

Link to publication record in Manchester Research Explorer

Citation for published version (APA):

Chen, H-J., Lanzon, A., \& Petersen, I. R. (2017). Conditions for preserving negative imaginary properties in feedback interconnections and an application to multi-agent systems. In Proceedings of the 25th Mediterranean Conference on Control and Automation, Valletta, Malta, Jul 2017 https://doi.org/10.1109/MED.2017.7984271

Published in:

Proceedings of the 25th Mediterranean Conference on Control and Automation, Valletta, Malta, Jul 2017

\section{Citing this paper}

Please note that where the full-text provided on Manchester Research Explorer is the Author Accepted Manuscript or Proof version this may differ from the final Published version. If citing, it is advised that you check and use the publisher's definitive version.

\section{General rights}

Copyright and moral rights for the publications made accessible in the Research Explorer are retained by the authors and/or other copyright owners and it is a condition of accessing publications that users recognise and abide by the legal requirements associated with these rights.

\section{Takedown policy}

If you believe that this document breaches copyright please refer to the University of Manchester's Takedown Procedures [http://man.ac.uk/04Y6Bo] or contact uml.scholarlycommunications@manchester.ac.uk providing relevant details, so we can investigate your claim.

\section{OPEN ACCESS}




\title{
Conditions for preserving negative imaginary properties in feedback interconnections and an application to multi-agent systems
}

\author{
Hsueh-Ju Chen ${ }^{1}$, Alexander Lanzon ${ }^{1}$, and Ian R. Petersen ${ }^{2}$
}

\begin{abstract}
We derived necessary and sufficient conditions for a feedback interconnection of negative imaginary systems to have negative imaginary properties. We first consider two negative imaginary systems (or strictly negative imaginary systems) which are interconnected via positive feedback. Then necessary and sufficient conditions for determining whether the feedback interconnection has negative imaginary properties are presented. These conditions are specialised to a dc loop gain that is easy to check under some assumptions. Together with stability results in the literature, these conditions could be applied to check the stability of a string of several coupled negative imaginary subsystems. Such an example is given to illustrate the usefulness of our proposed results.
\end{abstract}

\section{INTRODUCTION}

An important notion in control analysis and synthesis is the negative imaginary property. The concept of negative imaginary systems was firstly inspired by inertial systems [7]. Systems whose input and output are force and position (or acceleration) often can be modelled as negative imaginary systems. In the single-input, single-output case, the Nyquist plot of a negative imaginary system is below the real axis. Due to the special properties of negative imaginary systems, there have been wide practical applications in various areas, such as large space structures, nano-positioning control [1], [10], [13] and multi-agent networked systems [2], [20], [21]. The notion of negative imaginary systems was first presented in [7] and so was the negative imaginary lemma. This lemma is very useful for stability analysis and control synthesis of negative imaginary systems. Due of its relevance, the work is extended in [23] which considers negative imaginary systems having poles on the imaginary axis excluding the origin. A further extension in [11] allows negative imaginary systems to have free body dynamics.

Beyond the aforementioned development in negative imaginary lemmas and the negative imaginary notion, the theory has been specialised and extended also in several other directions. Here we mention some of them as follows. First, several modifications have generalised and specialised the set of the negative imaginary systems as presented in [7], such as discrete-time systems [4], non-rational systems [3], [5], strongly strict negative imaginary systems [8], infinite

\footnotetext{
${ }^{1}$ Hsueh-Ju Chen is with School of Electrical and Electronic Engineering, University of Manchester, Sackville Street, Manchester M13 9PL, U.K. hsueh-ju.chen@manchester.ac.uk

${ }^{1}$ Alexander Lanzon is with School of Electrical and Electronic Engineering, University of Manchester, Sackville Street, Manchester M13 9PL, U.K. Alexander. Lanzon@manchester.ac.uk

${ }^{2}$ Ian R. Petersen is with Research School of Engineering, Australian National University, Canberra, Australia ian . petersen@anu . edu . au
}

dimensional systems [14], Hamiltonian systems [19], descriptor systems [22], finite frequency negative imaginary systems [24], and lossless systems [25]. Second, various control synthesis approaches for negative imaginary systems can be found in [16], [17], [18]. Third, stability results for a positive feedback interconnection of a negative imaginary system and a strictly negative imaginary system are originally proposed in [7] and the results have been modified and applied to different classes of negative imaginary systems shown in [7], [9], [23], [24], [25]. Recently, [6] proposed generalised results that unify all the robust feedback stability work which appeared in earlier literature into one general theory. Fourth, [2] used the work in [7] and discussed the stability for a string of coupled strictly negative imaginary systems.

In this paper, we present necessary and sufficient conditions for negative imaginary properties to be preserved when considering two negative imaginary systems that are interconnected via positive feedback. To achieve this, according to the generalised definition of negative imaginary systems, we propose a negative imaginary lemma without minimality assumptions and this lemma can also be considered as extensions of the work in [11] and [17]. Then, based on the proposed negative imaginary lemma and several auxiliary lemmas, we derive necessary and sufficient conditions for preserving the negative imaginary properties of interconnected negative imaginary systems. The main results together with the stability results given in [6], [7], [23] are used to conclude the stability for a ring of coupled subsystems with negative imaginary dynamics. Unlike the results proposed in [2], which required the systems to be single input, single output, and strictly negative imaginary, our results can be applied to determine the stability for the interconnection of several subsystems without imposing the above restrictions. The main contributions of this work are thus: (i) derivation of a generalised negative imaginary lemma that relaxes the minimality assumptions; (ii) derivation of necessary and sufficient conditions for two strictly negative imaginary systems which are interconnected via positive feedback to remain within the same class of negative imaginary systems; (iii) derivation of generalised results for the positive feedback interconnection of two negative imaginary systems with poles on the imaginary axis except at the origin to preserve negative imaginary properties; (iv) under certain assumptions (i.e. strictly properness of a transfer function), the proposed main results specialise to simple and easy-to-check condition (i.e. dc loop gain condition); and (v) demonstration via a practical example to illustrate the usefulness of the derived 
work.

Notation: $\bar{\lambda}(A)$ is the largest eigenvalue of a square complex matrix that has only real eigenvalues. $\mathfrak{R}(a)$ is a real part of a complex number $a$. $\mathscr{R} \mathscr{H}_{\infty}$ denotes the set of real, rational, stable transfer function matrices.

\section{PRELIMINARY LEMMAS}

There are different notions of negative imaginary systems. Here, we recall the generalised definition of negative imaginary systems and this definition extends the ones proposed in [7] and [23] to include the case where negative imaginary systems could have possible poles on the imaginary axis and at the origin.

Definition 1: ([9]). Let $R(s)$ be a real, rational, proper transfer function. Then $R(s)$ is said to be Negative Imaginary (NI) if

1) $R(s)$ has no poles in $\mathfrak{R}(s)>0$;

2) $j\left[R(j \omega)-R(j \omega)^{*}\right] \geq 0$ for all $\omega \in(0, \infty)$ except the values of $\omega$ where $j \omega$ is a pole of $R(s)$;

$3)$ if $j \omega_{0}$ with $\omega_{0}>0$ is a pole of $R(s)$, then it is at most a simple pole and the residue matrix $K_{0}=\lim _{s \rightarrow j \omega_{0}}\left(s-j \omega_{0}\right) j R(s)$ is Hermitian and positive semidefinite;

4) if $s=0$ is a pole of $R(s)$, then $\lim _{s \rightarrow 0} s^{k} R(s)=0$ for all integer $k \geq 3$ and $\lim _{s \rightarrow 0} s^{2} R(s)$ is Hermitian and positive semidefinite.

We also recall the definition of strictly negative imaginary systems as follows.

Definition 2: ([7]). Let $R(s)$ be a real, rational, proper transfer function. Then $R(s)$ is said to be Strictly Negative Imaginary (SNI) if

1) $R(s)$ has no poles in $\mathfrak{R}(s) \geq 0$;

2) $j\left[R(j \omega)-R(j \omega)^{*}\right]>0$ for all $\omega \in(0, \infty)$.

Unlike negative imaginary systems which could have poles on the imaginary axis, strictly imaginary systems only allow systems to be stable. Besides, in the single-input, singleoutput case, the Nyquist plot of a negative imaginary system is below the real axis; therefore, the phase of the system must vary between $-\pi$ to 0 . Because of Definition 2, the Nyquist plot of strictly negative imaginary systems will never touch the real axis for $\omega \in(0, \infty)$.

Since in this paper we focus on systems which have negative imaginary properties, the negative imaginary lemma is used frequently for deviation of our main results. According to the literature, the first negative imaginary lemma was presented in [7] for stable negative imaginary systems and this result was extended in [23] for the case where negative imaginary systems have poles on the imaginary axis except at the origin; however, minimality assumptions are required in the lemma. Further modifications in [17] relaxed the minimality assumptions and in [11] allowed negative imaginary systems to have possible poles at the origin. Here, we built on these two results and present a generalised version of the negative imaginary lemma which is suitable for the case where negative imaginary systems could have possible poles at the origin without imposing minimality assumptions.
Lemma 3: Let $\left[\begin{array}{c|c}A & B \\ \hline C & D\end{array}\right]$ be a state-space realisation of $R(s)$.

(i) If $D=D^{T}$ and there exists a $P=P^{T} \geq 0$ such that

$$
\left[\begin{array}{cc}
P A+A^{T} P & P B-A^{T} C^{T} \\
B^{T} P-C A & -\left(C B+B^{T} C^{T}\right)
\end{array}\right] \leq 0,
$$

then $R(s)$ is negative imaginary.

(ii) If $R(s)$ is negative imaginary and its state-space realisation $\left[\begin{array}{l|l}A & B \\ \hline C & D\end{array}\right]$ has no observable uncontrollable modes, then $D=D^{T}$ and there exists a $P=P^{T} \geq 0$ satisfying (1).

Proof: The proof will be published elsewhere.

\section{INTERCONNECTED NEGATIVE IMAGINARY SYSTEMS VIA POSITIVE FEEDBACK}

In the literature, there are profound discussions on stability analysis for the positive feedback interconnection of negative imaginary systems. However, if we attempted to consider the stability for several subsystems with negative imaginary properties, which are interconnected via positive feedback, then the work mentioned previously may not be applicable. Reference [2] constrained systems to be single-input, singleoutput, strictly negative imaginary systems and derived the stability conditions for the coupling of several such subsystems. Unlike [2] which imposed restrictive assumptions, we here derive necessary and sufficient conditions for negative imaginary properties to be preserved when considering two interconnected multi-input, multi-output, negative imaginary systems with possible poles on the imaginary axis via positive feedback and together with the stability results proposed in [23] we establish the stability for a string of coupled negative imaginary subsystems which is illustrated via an example in the next section. Furthermore, if we imposed different and simple assumptions, the necessary and sufficient conditions for a feedback system to be a negative imaginary system presented in this paper simplifies as a dc loop gain condition that is a necessary and sufficient.

\section{A. Two strictly negative imaginary systems}

In this subsection, we consider the positive feedback interconnection of two strictly negative imaginary systems as shown in Fig. 1 and derived necessary and sufficient conditions for the feedback system to remain within the class of the negative imaginary systems that satisfies the conditions in Definition 2. The following theorem shows that negative imaginary properties can be carried over when we consider two strictly negative imaginary systems that are interconnected via positive feedback.

Theorem 4: Let $M(s)$ and $N(s)$ be strictly negative imaginary systems. Then the corresponding closed-loop transfer function from $\left[\begin{array}{l}w_{1} \\ w_{2}\end{array}\right]$ to $\left[\begin{array}{l}y_{1} \\ y_{2}\end{array}\right]$ denoted by $T(s)$ is a proper, strictly negative imaginary system if and only if 


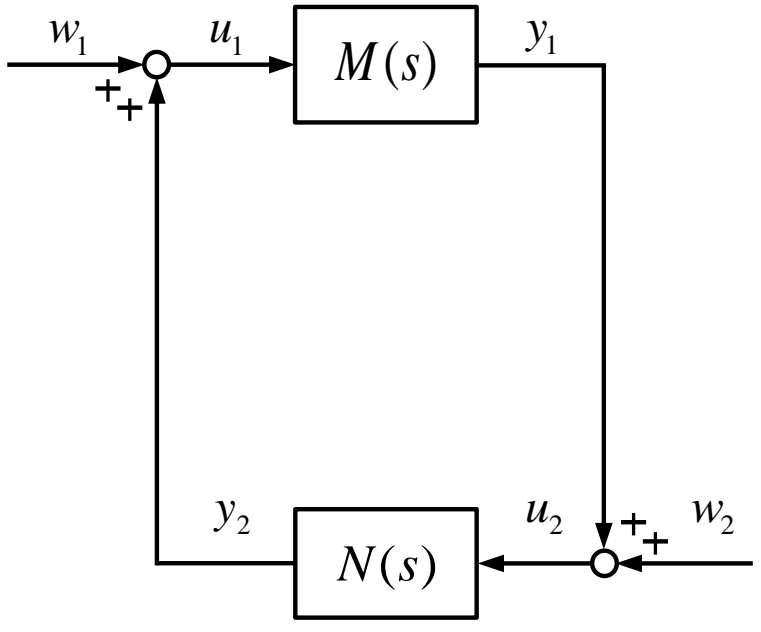

Fig. 1. Positive feedback interconnection of $M(s)$ and $N(s)$.

$$
\begin{aligned}
& I-M(\infty) N(\infty) \text { is nonsingular, } \\
& \bar{\lambda}\left[[I-M(\infty) N(\infty)]^{-1}(M(\infty) N(0)-I)\right]<0, \text { and } \\
& \bar{\lambda}\left[[I-N(0) M(\infty)]^{-1}(N(0) M(0)-I)\right]<0 .
\end{aligned}
$$

Proof: The proof will be published elsewhere.

It is easy to observe that the stability of the closed-loop transfer function $T(s)$ is guaranteed by the stability results proposed in [6]. Since $M(s)$ and $N(s)$ are strictly negative imaginary systems and $T(s)$ belongs to $\mathscr{R} \mathscr{H}_{\infty}$, we conclude that the feedback system is a strictly negative imaginary system by using [3, Theorem 6] which can be shown to hold true even for non-symmetric systems. Unlike the results proposed in [2] which only focused on systems with a single input and output, it is worth mentioning that this theorem and together with the stability results proposed in [6] could determine stability for the coupling of several multi-input, multi-output, strictly negative imaginary systems.

If we impose the assumption that $M(s)$ is strictly proper, a simple and easy-to-check condition for testing whether feedback system is a strictly negative imaginary system can be obtained.

Corollary 5: Let $M(s)$ and $N(s)$ be strictly negative imaginary systems. Also, suppose that $M(s)$ is strictly proper. Then the corresponding closed-loop transfer function from $\left[\begin{array}{l}w_{1} \\ w_{2}\end{array}\right]$ to $\left[\begin{array}{l}y_{1} \\ y_{2}\end{array}\right]$ denoted by $T(s)$ is a proper, strictly negative imaginary system if and only if $\bar{\lambda}[M(0) N(0)]<1$.

Proof: The proof will be published elsewhere.

The following example shows that the properties of strictly negative imaginary systems are carried over when we consider two strictly negative imaginary systems that are interconnected via positive feedback.

Example 1: Let us consider the positive feedback interconnection of two strictly negative imaginary systems $M(s)$ and $N(s)$ as shown in Fig. 1 where both $M(s)=\frac{1}{s+1}$ and $N(s)=\frac{-s}{s+1}$ are strictly negative imaginary systems. Also, $M(s)$ is strictly proper. Hence, the closed-loop transfer function matrix $T(s)$ from $\left[\begin{array}{l}w_{1} \\ w_{2}\end{array}\right]$ to $\left[\begin{array}{l}y_{1} \\ y_{2}\end{array}\right]$ is a proper, strictly negative imaginary system via Corollary 5 because $\bar{\lambda}[M(0) N(0)]=0<1$ under the assumption that $M(s)$ is strictly proper. In fact, $T(s)=\frac{1}{s^{2}+3 s+1}\left[\begin{array}{cc}s+1 & -s \\ -s & -s^{2}-s\end{array}\right]$ which can be shown to fulfill the required properties.

A different way of simplifying the conditions given in Theorem 4 is to impose two instantaneous gain assumptions as imposed in earlier literature such as [6]. Then the condition obtained is the same as the one in the previous corollary in which a dc gain condition is necessary and sufficient.

Corollary 6: Let $M(s)$ and $N(s)$ be strictly negative imaginary systems. Suppose $M(\infty) N(\infty)=0$ and $N(\infty) \geq 0$. Then the corresponding closed-loop transfer function from $\left[\begin{array}{l}w_{1} \\ w_{2}\end{array}\right]$ to $\left[\begin{array}{l}y_{1} \\ y_{2}\end{array}\right]$ denoted by $T(s)$ is a proper, strictly negative imaginary system if and only if $\bar{\lambda}[M(0) N(0)]<1$.

Proof: The proof will be published elsewhere.

B. Two negative imaginary systems without poles at the origin

As we mentioned in the previous subsection, a number of conditions need to be fulfilled to make a feedback system have strictly negative imaginary properties. Here, based on earlier work [12] we present necessary and sufficient conditions for the positive feedback interconnection of negative imaginary systems with poles on the imaginary axis to preserve the negative imaginary properties. This result is given in the following theorem.

Theorem 7: Let $M(s)$ and $N(s)$ be negative imaginary systems without poles at the origin. Then the corresponding closed-loop transfer function from $\left[\begin{array}{l}w_{1} \\ w_{2}\end{array}\right]$ to $\left[\begin{array}{l}y_{1} \\ y_{2}\end{array}\right]$ denoted by $T(s)$ is proper, negative imaginary, and has no poles at the origin (resp. $\operatorname{det}(I-M(\infty) N(0)) \neq 0$ ) if and only if

$$
\begin{aligned}
& I-M(\infty) N(\infty) \text { is nonsingular, } \\
& \bar{\lambda}\left[[I-M(\infty) N(\infty)]^{-1}(M(\infty) N(0)-I)\right]<0, \text { and } \\
& \bar{\lambda}\left[[I-N(0) M(\infty)]^{-1}(N(0) M(0)-I)\right]<0 \text { (resp. } \leq
\end{aligned}
$$
$0)$.

Proof: The proof will be published elsewhere.

Remark 8: Under the above conditions, interconnected negative imaginary systems without free body motion can be made negative imaginary or negative imaginary system without poles at the origin. Negative imaginary with possible poles at the origin require the condition with non-strict inequality in the third condition (i.e. $\bar{\lambda}[[I-$ $\left.\left.N(0) M(\infty)]^{-1}(N(0) M(0)-I)\right] \leq 0\right)$ which can be directly seen from Theorem 7. For the conditions which include the non-strict inequality in the last condition, the matrix $P$ that satisfies the LMI condition in (1) is no longer a positive definite matrix but a positive-semidefinite matrix; hence, instead of using the negative imaginary lemma proposed in [23, Lemma 7], one can use Lemma 3(i) to show that the system is negative imaginary with possible poles at the origin. On the other hand, $\operatorname{det}(I-M(\infty) N(0)) \neq 0$ is required because a Schur complement can be used to ensure that the three conditions in Theorem 7 are indeed fulfilled. 
Another way of simplifying the conditions in Theorem 7 is to assume that the transfer function matrix $M(s)$ is strictly proper. The condition to make a feedback system have negative imaginary properties is simple and compact.

Corollary 9: Let $M(s)$ and $N(s)$ be negative imaginary systems without poles at the origin. Also, suppose that $M(s)$ is strictly proper. Then the corresponding closed-loop transfer function from $\left[\begin{array}{l}w_{1} \\ w_{2}\end{array}\right]$ to $\left[\begin{array}{l}y_{1} \\ y_{2}\end{array}\right]$ denoted by $T(s)$ is a proper, negative imaginary system without poles at the origin (resp. proper, negative imaginary system) if and only if $\bar{\lambda}[M(0) N(0)]<1$ (resp. $\leq 1)$.

Proof: The proof will be published elsewhere.

Example 2 illustrates that an interconnection of two negative imaginary systems without free body motion via positive feedback does have negative imaginary frequency response via the proposed corollary.

Example 2: Let us consider the positive feedback interconnection of two negative imaginary systems $M(s)$ and $N(s)$ as shown in Fig. 1 where both $M(s)=\frac{1}{s^{2}+1}$ and $N(s)=\frac{1}{s^{2}+1}$ are negative imaginary systems without poles at the origin. Also, $M(s)$ is strictly proper. Hence, since $M(s)$ is strictly proper, the closed-loop transfer function matrix $T(s)$ from $\left[\begin{array}{l}w_{1} \\ w_{2}\end{array}\right]$ to $\left[\begin{array}{l}y_{1} \\ y_{2}\end{array}\right]$ is a proper, negative imaginary system via Corollary 9 because $\bar{\lambda}[M(0) N(0)]=$ $1 \leq 1$. In fact, $T(s)=\frac{1}{s^{4}+2 s^{2}}\left[\begin{array}{cc}s^{2}+1 & 1 \\ 1 & s^{2}+1\end{array}\right]$ which can be shown to have the required properties.

\section{EXAmple: Decentralised Control of Vehicle PLATOONS}

It was stated in the previous section that under specific conditions, interconnected negative imaginary systems will have negative imaginary properties. Here, we use a practical example to illustrate the main results and this example is directly taken from [2] which focused on strictly negative imaginary systems with a single input and output. However, here we consider each subsystem as a negative imaginary system with possible poles on imaginary axis except at the origin. Then, we apply our proposed results and the stability results presented in the literature [23] to demonstrate that the system is stabilized via decentralised control.

We consider five vehicles cruising in a straight line and the dynamic equation of each vehicle can be expressed as double integrator:

$$
\ddot{y}_{i}=u_{i} \forall i=1,2, \ldots, 5
$$

where $y_{i}$ denotes the position of the $i$ th vehicle and $u_{i}$ is the torque which is applied to the $i$ th vehicle. The control strategy proposed in [2] is used to maintain a distance $L>0$ between two adjacent vehicles and achieve a desired travelling speed $V>0$ for each vehicle. The input of the system is chosen as follows:

$u_{1}=-\xi_{1}\left(\dot{y}_{1}(t)-V\right)-F_{1}\left(y_{1}(t)-V t+L\right)+B_{1} \delta_{2}(t)$, $u_{i}(t)=-\xi_{i}\left(\dot{y}_{i}(t)-V\right)-F_{i} \delta_{i}(t)+B_{i} \delta_{i+1}(t) \forall i=$ 2,3 , and 4 ,

$$
u_{5}(t)=-\xi_{5}\left(\dot{y}_{5}(t)-V\right)-F_{5} \delta_{5}(t)
$$

where $\delta_{i}(t)=y_{i}(t)-y_{i-1}(t)+L, \xi_{i}$ denotes the damping parameter and $F_{i}$ and $B_{i}$ are control gains of the $i$ th vehicle. For simplicity, we assume $x_{i}(t)=y_{i}(t)-V t+i L$. Via a change of variables, the transformed system can be expressed as

$$
\begin{aligned}
& \ddot{x}_{1}+\xi_{1} \dot{x}_{1}=-F_{1} x_{1}-B_{1}\left(x_{1}-x_{2}\right), \\
& \ddot{x}_{i}+\xi_{i} \dot{x}_{i}=-F_{i}\left(x_{i}-x_{i-1}\right)-B_{i}\left(x_{i}-x_{i+1}\right), \\
& \forall i=2,3, \text { and } 4, \\
& \ddot{x}_{5}+\xi_{5} \dot{x}_{5}=-F_{5}\left(x_{5}-x_{4}\right) .
\end{aligned}
$$

The whole system with control input as shown in (2) can be represented as a block diagram as in Fig. 2 where each subsystem

$$
\begin{aligned}
& G_{i}(s)=\frac{1}{s^{2}+\xi_{i} s+\left(B_{i}+F_{i}\right)} \forall i=1,2, \ldots, 4, \text { and } \\
& G_{5}(s)=\frac{1}{s^{2}+\xi_{5} s+F_{5}} .
\end{aligned}
$$

Besides, let $\Phi_{i}(s)$ be the transfer function of the positive feedback interconnection of the first $i+1$ subsystems, and via simple calculation we obtain that

$$
\begin{aligned}
& \Phi_{1}(s)=\frac{G_{2}(s)}{1-B_{1} F_{2} G_{1}(s) G_{2}(s)}, \text { and } \\
& \Phi_{i}(s)=\frac{G_{i+1}(s)}{1-B_{i} F_{i+1} \Phi_{i-1}(s) G_{i+1}(s)} \text { for } i=2 \text { and } 3 .
\end{aligned}
$$

If we set gain $F_{i}=1 \forall i=1,2, \ldots, 5$, the parameters $\xi_{i}=0$ and gain $B_{i}=1$ for $i=1,2,3$, and 4 , and $\xi_{5}=1$, then $G_{i}(s) \forall i=1,2, \ldots, 4$ are negative imaginary systems without free body motion and $G_{5}(s)$ is a strictly negative imaginary system via [23, Definition 1] and Definition 2 respectively. Since under the assumptions both $G_{1}(s)$ and $G_{2}(s)$ are negative imaginary systems with $G_{2}(\infty)=0$ and the dc loop gain is less than 1 , we conclude that the $2 \times 2$ closed-loop transfer function from $\left(w_{1}, w_{2}\right)$ to $\left(y_{1}, y_{2}\right)$ denoted by $T(s)$ in Figure 1 is a negative imaginary system without poles at the origin via Corollary 9. Hence, $\Phi_{1}(s)$, which is the $(2,2)$ element of $T(s)$, is in the same class of negative imaginary systems. Through the same procedure, we know that $\Phi_{2}(s)$ and $\Phi_{3}(s)$ also belong to the same class. Finally, we rearrange the control system in Fig. 2 into the block diagram as in Fig. 3 and apply the stability results from [6, Corollary 12] to show that the applied control approach does stabilise the vehicle platoon.

\section{CONCLUSION}

This paper generalises previous results and derives necessary and sufficient conditions for a feedback system to have negative imaginary properties. When we consider a positive feedback interconnection of two negative imaginary systems, under some assumptions, conditions for the negative imaginary properties to be preserved are derived. Conditions for a closed-loop system to be negative imaginary or negative imaginary without poles at the origin are slightly different and the reason for that is also discussed. Then, these main results are specialised into a simple and compact condition (i.e. a dc loop gain condition) under specific assumptions. The main results allow for negative imaginary systems with possible poles on the imaginary axis and are suitable for not only single-input, single-output cases but also multiinput, multi-output cases. Internal stability is not a required 


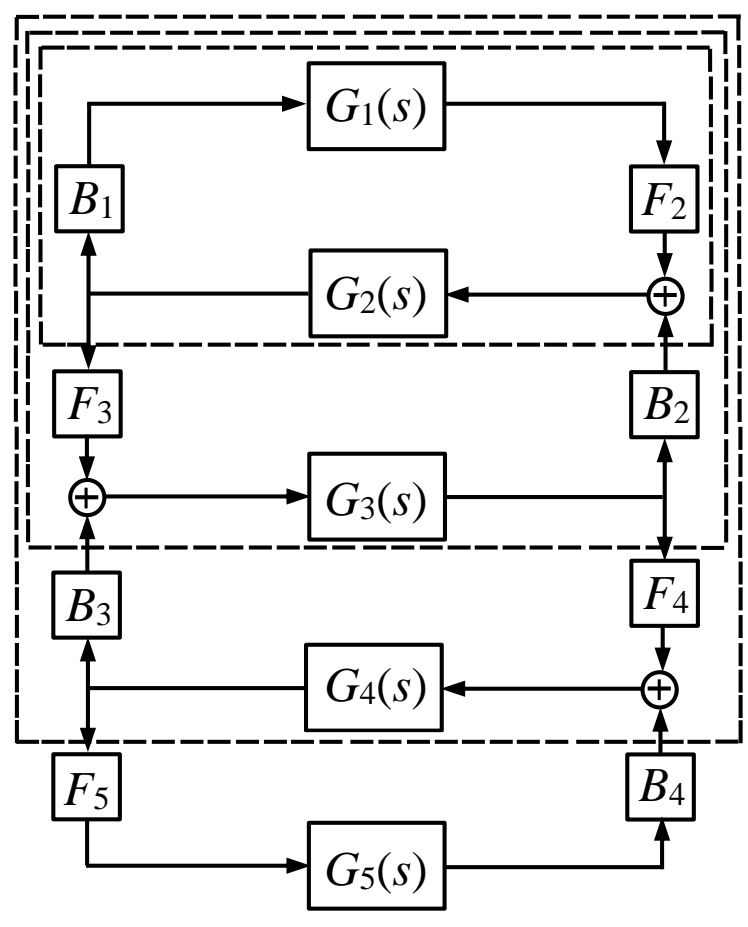

Fig. 2. Decentralised control of vehicle platoons.

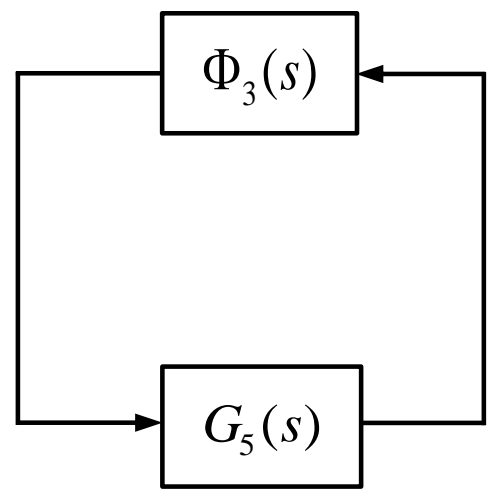

Fig. 3. Rearranged control system.

assumption to obtain closed-loop negative imaginary properties when negative imaginary systems are interconnected in positive feedback, unlike restrictions imposed in earlier literature (e.g. [12, Theorem 1] and [15, Theorem 2]). Last, we applied our main results, together with prior stability results, to the decentralised control of vehicle platoons and the results did show that the whole system can be stabilised by the presented control strategy. Possible future work could be done by extending this work to allow the negative imaginary systems to include the poles at the origin.

\section{REFERENCES}

[1] B. Bhikkaji, S. O. R. Moheimani, and I. R. Petersen. A negative imaginary approach to modeling and control of a collocated structure. IEEE/ASME Transactions on Mechatronics, 17(4):717-727, 2012.

[2] C. Cai and G. Hagen. Stability analysis for a string of coupled stable subsystems with negative imaginary frequency response. IEEE Transactions on Automatic Control, 55(8):1958-1963, 2010.
[3] A. Ferrante, A. Lanzon, and L. Ntogramatzidis. Foundations of not necessarily rational negative imaginary systems theory: Relations between classes of negative imaginary and positive real systems. IEEE Transactions on Automatic Control, 61(10):3052-3057, 2016.

[4] A. Ferrante, A. Lanzon, and L. Ntogramatzidis. Discrete-time negative imaginary systems. Automatica, 79:1-10, 2017.

[5] A. Ferrante and L. Ntogramatzidis. Some new results in the theory of negative imaginary systems with symmetric transfer matrix function. Automatica, 49(7):2138-2144, 2013.

[6] A. Lanzon and H. J. Chen. Feedback stability of negative imaginary systems. IEEE Transactions on Automatic Control. In press, DOI: 10.1109/TAC.2017.2688579.

[7] A. Lanzon and I. R. Petersen. Stability robustness of a feedback interconnection of systems with negative imaginary frequency response. IEEE Transactions on Automatic Control, 53(4):1042-1046, 2008.

[8] A. Lanzon, Z. Song, S. Patra, and I. R. Petersen. A strongly strict negative-imaginary lemma for non-minimal linear systems. Соттиnications in Information and Systems, 11(2):139-152, 2011.

[9] M. A. Mabrok, A. G. Kallapur, I. R. Petersen, and A. Lanzon. Generalizing negative imaginary systems theory to include free body dynamics: Control of highly resonant structures with free body motion. IEEE Transactions on Automatic Control, 59(10):2692-2707, 2014.

[10] M. A. Mabrok, A. G. Kallapur, I. R. Petersen, and A. Lanzon. Spectral conditions for negative imaginary systems with applications to nanopositioning. IEEE/ASME Transactions on Mechatronics, 19(3):895903, 2014.

[11] M. A. Mabrok, A. G. Kallapur, I. R. Petersen, and A. Lanzon. A generalized negative imaginary lemma and Riccati-based static statefeedback negative imaginary synthesis. Systems and Control Letters, 77:63-68, 2015.

[12] M. A. Mabrok and I. R. Petersen. Negative imaginary feedback systems. In Proceedings of the 4th Australian Control Conference, pages 130-133, Canberra, Australia, 2014.

[13] I. A. Mahmood, S. O. R. Moheimani, and B. Bhikkaji. A new scanning method for fast atomic force microscopy. IEEE Transactions on Nanotechnology, 10(2):203-216, 2011.

[14] M. R. Opmeer. Infinite-dimensional negative imaginary systems. IEEE Transactions on Automatic Control, 56(12):2973-2976, 2011.

[15] I. R. Petersen and A. Lanzon. Feedback control of negative-imaginary systems. IEEE Control Systems Magazine, 30(5):54-72, 2010.

[16] Z. Song, A. Lanzon, S. Patra, and I. R. Petersen. Towards controller synthesis for systems with negative imaginary frequency response. IEEE Transactions on Automatic Control, 55(6):1506-1511, 2010.

[17] Z. Song, A. Lanzon, S. Patra, and I. R. Petersen. A negativeimaginary lemma without minimality assumptions and robust statefeedback synthesis for uncertain negative-imaginary systems. Systems and Control Letters, 61(12):1269-1276, 2012.

[18] Z. Song, A. Lanzon, S. Patra, and I. R. Petersen. Robust performance analysis for uncertain negative-imaginary systems. International Journal of Robust and Nonlinear Control, 22(3):262-281, 2012.

[19] A. J. van der Schaft. Positive feedback interconnection of Hamiltonian systems. In Proceedings of the 50th IEEE Conference on Decision and Control and European Control Conference, pages 6510-6515, Orlando, FL, USA, 2011.

[20] J. Wang, A. Lanzon, and I. R. Petersen. Robust cooperative control of multiple heterogeneous negative-imaginary systems. Automatica, 61:64-72, 2015.

[21] J. Wang, A. Lanzon, and I. R. Petersen. Robust output feedback consensus for networked negative-imaginary systems. IEEE Transactions on Automatic Control, 60(9):2547-2552, 2015.

[22] J. Xiong, A. Lanzon, and I. R. Petersen. Negative imaginary lemmas for descriptor systems. IEEE Transactions on Automatic Control, 61(2):491-496, 2016.

[23] J. Xiong, I. R. Petersen, and A. Lanzon. A negative imaginary lemma and the stability of interconnections of linear negative imaginary systems. IEEE Transactions on Automatic Control, 55(10):2342-2347, 2010.

[24] J. Xiong, I. R. Petersen, and A. Lanzon. Finite frequency negative imaginary systems. IEEE Transactions on Automatic Control, 57(11):2917-2922, 2012.

[25] J. Xiong, I. R. Petersen, and A. Lanzon. On lossless negative imaginary systems. Automatica, 48(6):1213-1217, 2012. 